\title{
Monte Carlo Simulation and Experimental High-Angle Annular Dark Field Tomography
}

\author{
Frédéric Voisard, Nicolas Brodusch, Hendrix Demers, and Raynald Gauvin
}

Department of Mining and Materials Engineering, McGill University, Montréal, Québec, Canada.

In the last 10 years, the transmission electron tomography technique was extensively used in biology applications to obtain 3D configurations of small organic structures [1]. Such bright field electron tomography is not generally used in material science applications as the diffraction effects result in strong artefacts during the reconstruction. Bragg's diffractions are indeed present at certain angles during the acquisition of a bright field tilt series. High angle annular dark field (HAADF) imaging with a scanning transmission electron microscope (STEM) is not significantly affected by dynamic effects. Since the contrast of HAADF depends on material thickness and composition [2], this technique is a good candidate for tomographic acquisitions of materials science samples. However, the reconstruction algorithms have to be adapted and optimum experimental parameters are needed for high resolution three-dimensional (3D) dataset at the nanoscale. The CASINO Monte Carlo software [3] was developed to simulate the effect of parameters such as angular tilt range, angular coverage, beam energy and beam dosage on HAADF-STEM micrograph [4]. In this work, we use a Monte Carlo-based simulation method to accurately model the whole tomographic data acquisition process and test the reconstruction algorithm with these different parameters.

The Monte Carlo simulation software CASINO [3] was used to simulate a HAADF tomography tilt series. The virtual sample in Figure 1 was composed of a 114 titania $\left(\mathrm{TiO}_{2}\right)$ rutile nanoparticles (NPs) modeled by spheres of 7 to $9 \mathrm{~nm}$ in diameter. The ELSESPA elastic cross section model [5] is used for the simulations of micrograph of $93 \times 75$ pixels, with a probe size of $0.2 \mathrm{~nm}$ and a pixel size of $1 \mathrm{~nm}$. High angle scattering is defined as electrons scattered at angled between 94 and 611 mrad. Figure 1 shows the effect of different acquisition schemes on the reconstruction results. The bright and dark lines and the elongated shape of the NPs are artefacts created by the reconstruction method, because of the lack of angular coverage. Fewer artefacts were observed when the tilt range was increased or with smaller tilt interval.

A preliminary experimental tilt series was performed on a titania $\left(\mathrm{TiO}_{2}\right)$ rutile nanoparticles sample. The sample is prepared by controlled precipitation of titanium oxide out of titanium tetrachloride solution. The nanoparticles have a natural tendency to form aggregates. An aggregate was imaged at $110 \mathrm{kX}$, for a pixel size of $1.7 \mathrm{~nm}$. The tilt series was acquired from $\pm 60^{\circ}$, with a tilt step of $2^{\circ}$. Figure 2 shows the 3D dataset after reconstruction. The experimental reconstruction was not able to clearly separate the NPs, but the Monte Carlo simulation results showed that the experimental parameters used were not optimum for this sample. More parameters will be simulated and their effect on the reconstruction will be analysed, than used experimentally to obtain a high resolution 3D dataset of titania $\left(\mathrm{TiO}_{2}\right)$ rutile nanoparticles.

\section{References:}

[1] J. Frank in "Electron tomography: methods for three-dimensional visualization of structures in the cell”, 2nd ed. (Springer, New York), p.1. 
[2] DB. Williams "Transmission electron microscopy: a textbook for materials science" 2nd ed. (New York, Springer) p.39.

[3] Demers H et al, Scanning 33 (2011), p. 135-46.

[4] Demers Het al, Microscopy and Microanalysis 162010 p. 795-804.

[5] Salvat F et al, Computer Physics Communications 1652005 p.157-190.

[6] Messaoudi C et al. BMC Bioinformatics 82007 p.288

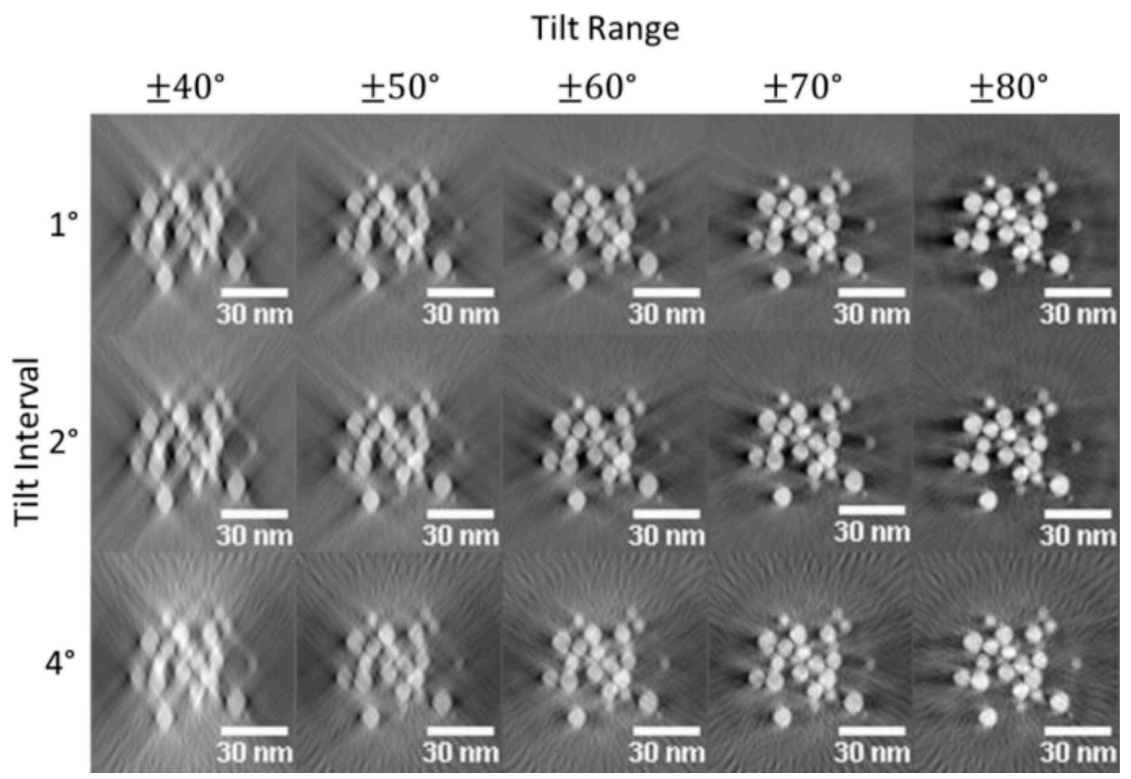

Figure 1. Comparison of tilt series acquisition for high-angle annular dark field tomography of an aggregate of titania $\left(\mathrm{TiO}_{2}\right)$ rutile nanoparticles. The simulated tilt series micrographs were obtained by Monte Carlo simulation [3] and the reconstruction performed using TOMOJ [4], with a varying tilt range and tilt interval.
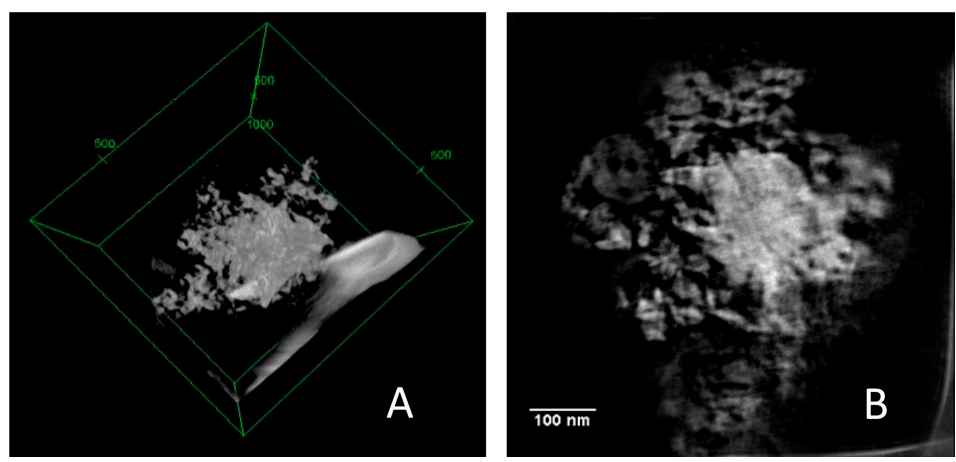

Figure 2. High-angle annular dark field (HAADF) tomography of an aggregate of titania $\left(\mathrm{TiO}_{2}\right)$ rutile nanoparticles. The experimental tilt series micrograph were obtained using a FEI Tecnai $\mathrm{G}^{2}$ F20 STEM microscope, using the HAADF detector and The reconstruction is performed using TOMOJ's [4] weighted back-projection algorithm [6], with a weighting factor of 93 and a weighting diameter of 512 pixels. A) 3D dataset rendering of the titania aggregate. B) A slice through the reconstructed volume. 